\title{
Interaction of Wilson loops in a confining vacuum
}

\author{
V. I. Shevchenko* \\ Institute for Theoretical Physics, Utrecht University, Utrecht, the Netherlands \\ and Institute of Theoretical and Experimental Physics, Moscow, Russia \\ Yu. A. Simonov ${ }^{\dagger}$ \\ Jefferson Laboratory, Newport News, Virginia 23606 \\ Institute of Theoretical and Experimental Physics, Moscow, Russia \\ (Received 23 May 2002; published 30 September 2002)
}

\begin{abstract}
Nonperturbative and perturbative interaction mechanisms of Wilson loops are studied within the background field formalism. The first one operates when the distance between the minimal surfaces of the loops is small and may be important for sea quark effects and strong decay processes. The second mechanism—the perturbative interaction in a nonperturbative confining background-is found to be physically dominant for all loop configurations characteristic of scattering processes. It reduces to perturbative gluon exchanges at small distances, while at larger distances it corresponds to the $t$-channel exchange of glueball states. A comparison to other approaches is made and the possible physical applications are discussed.
\end{abstract}

DOI: 10.1103/PhysRevD.66.056012

PACS number(s): 11.15. $-\mathrm{q}$

\section{INTRODUCTION}

The interaction of Wilson loops in QCD is the basic element of many physical applications. One can mention the hadron-hadron scattering amplitude, in particular the phenomenology of Pomeron exchange; the assumed color transparency phenomenon, strong hadron decays and OkuboZweig-Iizuka- (OZI-) forbidden processes, etc. There is also considerable interest in calculating Wilson loop and Polyakov loop correlators per se, not only in QCD but also in other field theories, in particular in supersymmetric YangMills theory. In all cases one starts with the connected average of two (or more) Wilson loops and tries to calculate it in the region of interest using the appropriate field-theoretical technique. It is the aim of the present paper to do this in the framework of the field correlator method in gluodynamics (see, e.g., the review [1] and references therein) incorporating both perturbative and nonperturbative contributions. Let us briefly recall the basic ideas behind the method. The general Wilson loop approach was introduced originally for heavy quarkonia [2]. For quarks of finite mass one can use the Feynman-Schwinger representation (see [3] for a review and references therein) to write the meson Green's function as an integral over all possible Wilson loops formed by the quark trajectories and finally to express the meson (and baryon) dynamics in terms of gauge-invariant correlators of the field strengths, characterizing the properties of the confining background. When going to the hadron-hadron scattering one can adopt the same formalism to express the scattering amplitude through the vacuum average of the product of two Wilson loops, with the subsequent integration over the ensemble of loops. To proceed, we use the background field formalism to separate nonperturbative gluon configurations from perturbative (sometimes called "valence") gluons.

\footnotetext{
*Email address: V.Shevchenko@phys.uu.nl

${ }^{\dagger}$ Email address: simonov@heron.itep.ru
}

Thus the answer will contain two parts: purely nonperturbative and perturbative inside a nonperturbative background, i.e., glueball exchanges between Wilson loops.

For the former it is convenient to use the non-(Abelian) Stokes theorem and express the answer in terms of the gauge-invariant field correlators and finally via the string tension. The perturbative part in a nonperturbative background physically corresponds to an exchange of glueball states between loops. We shall keep the number of colors $N_{c}$ as a free parameter in what follows. It will be argued that the leading term for a typical kinematics of the scattering process is the (background-modified) perturbative one. This is in line with the old observation that the high-energy scattering amplitude is dominated by the Pomeron exchange. We do not consider here the leading in $1 / N_{c}$ terms of ordinary Reggeon exchanges, which formally refer to the one-loop case and are subleading in the high-energy limit. We are also not discussing pion exchanges which may give the main contribution in some cases at not large energies; we will concentrate our attention on the case of the theory without dynamical quarks, i.e., gluodynamics. In another physical situation, e.g., when accounting for the sea quark loops or for a decay transition of a hadron state, the roles of the perturbative and nonperturbative mechanisms may change depending on the hadron quantum numbers. In all cases, however, the loop-loop interaction is the starting point of the field correlator formalism application for scattering, strong decay, etc. In Sec. II we introduce the general background formalism for the interaction of Wilson loops. In Sec. III the nonperturbative mechanism is studied in detail. In Sec. IV perturbative gluon exchange is shown to transform into the glueball exchange mechanism at large distances. Section V is devoted to a physical discussion of the results and a brief comparison with the existing models.

\section{INTERACTION OF WILSON LOOPS IN THE BACKGROUND FIELD FORMALISM}

In this section we are going to exploit the background field formalism [4] in the form worked out in [5]. We refer 
the interested reader to the cited papers for all the details and recall the basic steps only briefly. We start with decomposing the gluon field $A_{\mu}(x)$ into a nonperturbative background $B_{\mu}(x)$ and a perturbative part $a_{\mu}$, propagating in the background:

$$
A_{\mu}=B_{\mu}+a_{\mu} .
$$

The total gauge transformation is decomposed as

$$
B_{\mu} \rightarrow U^{\dagger}\left(B_{\mu}-\frac{i}{g} \partial_{\mu}\right) U, \quad a_{\mu} \rightarrow U^{\dagger} a_{\mu} U .
$$

The principle of separation is of no importance at the level of the partition function due to the obvious identity

$$
\begin{aligned}
Z & =\frac{1}{\mathcal{N}} \int \mathcal{D} A_{\mu} \exp (-S[A]) \\
& =\frac{1}{\mathcal{N}^{\prime}} \int \mathcal{D} B_{\mu} \int \mathcal{D} a_{\mu} \exp (-S[B+a])
\end{aligned}
$$

(here gauge-fixing and ghost terms are assumed to be included in the measure of integration). The Wilson loop depends on both $B_{\mu}$ and $a_{\mu}$ :

$$
\begin{aligned}
W(C)= & \frac{1}{N_{c}} \operatorname{TrP} \exp \left(i g \int_{C}\left(B_{\mu}^{c}+a_{\mu}^{c}\right) t^{c} d z_{\mu}\right) \\
= & \frac{1}{N_{c_{M}}} \lim _{\min } \operatorname{Tr} \prod_{m=1}^{M} \mathrm{P}\left\{1+i g\left[B_{\mu}\left(z^{[m]}\right)\right.\right. \\
& \left.\left.+a_{\mu}\left(z^{[m]}\right)\right] \Delta z_{\mu}^{[m]}\right\} .
\end{aligned}
$$

The trace in fundamental representation is normalized as

$$
\operatorname{Tr} \hat{\mathbf{1}}=N_{c}, \quad \operatorname{Tr} t^{a} t^{b}=\frac{1}{2} \delta^{a b} .
$$

Our general strategy is the following [5]: we expand the correlators under study in powers of the field $a_{\mu}$, while accounting for effects caused by the nonperturbative background exactly (i.e., without expansion in powers of $B_{\mu}$ ); namely, one has

$$
W(C)=W^{(0)}(C)+W^{(1)}(C)+W^{(2)}(C)+\cdots
$$

where, e.g.,

$$
\begin{aligned}
W^{(1)}(C)= & \frac{i g}{N_{c}} \operatorname{TrP}_{z u} \int_{C} a_{\mu}(z) d z_{\mu} \\
& \times \exp \left(i g \int_{C_{z}} B_{\nu}(u) d u_{\nu}\right),
\end{aligned}
$$

while $W^{(0)}(C)$ contains only the field $B_{\mu}$ and the ordering operator $\mathrm{P}_{z u}$ takes care of the ordering $a_{\mu}(z)$ and $B_{\mu}(u)$.

Let us define now the connected average of two Wilson loops as

$$
\chi\left(C_{1}, C_{2}\right)=\left\langle W\left(C_{1}\right) W\left(C_{2}\right)\right\rangle-\left\langle W\left(C_{1}\right)\right\rangle\left\langle W\left(C_{2}\right)\right\rangle .
$$

This average can also be expanded in powers of $g a_{\mu}$ :

$$
\begin{aligned}
\chi\left(C_{1}, C_{2}\right)= & \chi^{(0)}\left(C_{1}, C_{2}\right)+\chi^{(2)}\left(C_{1}, C_{2}\right) \\
& +\chi^{(4)}\left(C_{1}, C_{2}\right)+\cdots .
\end{aligned}
$$

Here $\chi^{(0)}\left(C_{1}, C_{2}\right)$ is the purely nonperturbative interaction of two Wilson loops and depends only on the fields $B_{\mu}$, while higher terms $\chi^{(n)}\left(C_{1}, C_{2}\right)$ are proportional to the average of $\left(g a_{\mu}\right)^{n}$. One immediately notices that since $\left\langle W^{(1)}\right\rangle$ is identically zero the term $\chi^{(2)}\left(C_{1}, C_{2}\right)$ vanishes and the expansion starts with the two-gluon exchange term $\chi^{(4)}\left(C_{1}, C_{2}\right)$.

In some cases the $C$-odd exchange contribution (odderon type) is also important; it is contained in $\chi^{(6)}\left(C_{1}, C_{2}\right)$. In what follows we discuss mostly the purely nonperturbative term $\chi^{(0)}\left(C_{1}, C_{2}\right)$, and in the last part of the paper also the two-gluon exchange $\chi^{(4)}\left(C_{1}, C_{2}\right)$ in Sec. IV.

\section{NONPERTURBATIVE INTERACTION OF WILSON LOOPS}

We consider in this section the first term in the expansion (8), namely, $\chi^{(0)}\left(C_{1}, C_{2}\right)$, and use the contour gauge [6] to write down the Wilson loop as a surface integral:

$$
W(C)=\frac{1}{N_{c}} \operatorname{Tr}, \mathrm{P} \exp \left(i g \int_{S} F_{\mu \nu}\left(u, x_{0}\right) d \sigma_{\mu \nu}(u)\right) .
$$

We have defined in Eq. (9) $F_{\mu \nu}\left(u, x_{0}\right)=\Phi_{L_{x_{0} u}} F_{\mu \nu}(u) \Phi_{L_{u x_{0}}}$ where the phase factors along the curve $L_{x_{0} u}$ with the edge points $x_{0}$ and $u$ are given by

$$
\Phi_{L_{x_{0} u}}=\mathrm{P} \exp \left(i g \int_{u}^{x_{0}} B_{\mu}(z) d z \mu\right) .
$$

Consider Wilson loops defined for two contours $C_{1}$ and $C_{2}$, where individual minimal surfaces will be denoted as $S_{1}^{\text {min }}$ and $S_{2}^{\min }$, respectively, throughout the paper. ${ }^{1}$ The typical problem in the discussed framework is to choose optimal integration surfaces in integrals of the form (9). The Wilson loop (9) is gauge invariant and surface independent. For a single Wilson loop it can be argued (see discussion and references in [1,7]) that in the case of a minimal surface (which is obviously the distinguished surface for a given contour) the dominant nonperturbative contribution in the cluster expansion of Eq. (9) comes from the lowest, Gaussian correlator of the field strength operators. This property is known as Gaussian dominance [8] and it plays an important role in all phenomenological applications of the field correlator method.

In the physical picture described above the appearance of the minimal surface has been in some sense a result of the

\footnotetext{
${ }^{1}$ For any surface we use one and the same letter $S$ for a surface as geometrical object and for its area.
} 
field correlator dynamics. However, one can take another view by saying that Gaussian dominance corresponds to that profile of the confining string worldsheet that minimizes the total energy of the system. In other words, to calculate $\langle W(C)\rangle$ one may proceed as follows: First, find the minimal energy of the confining string configuration (trivially corresponding to the minimal area surface in the case of a single static loop) and, as the next step, calculate the average (9) with a Gaussian ensemble of correlators integrated over this surface. It is this principle which we shall use in what follows to choose the shape of the surfaces entering our problem. For example, the Green's function describing an interacting two-meson system

$$
G^{[4]}=\left\langle\Psi_{L_{x \bar{x}}}^{\dagger} \Psi_{L_{u \bar{u}}}^{\dagger} \Psi_{L_{y \bar{y}}} \Psi_{L_{v \bar{v}}}\right\rangle
$$

where

$$
\Psi_{L_{y \bar{y}}}=\phi^{\dagger}(y) \Phi_{L_{y y}} \phi(\bar{y})
$$

and $\phi(x), \Phi_{L_{y y}-}$ represent quark fields and phase factors along the contour $L_{y \bar{y}}$, respectively, must be dominated at large separation/time by the confining string configuration of lowest total energy, in direct analogy with the case of a single meson.

It should be stressed from the very beginning that in the approach adopted by us in the present paper the confining strings are assumed to be infinitely thin. Correspondingly, we do not study the effects of string overlap, and assume that for two well separated loops the minimal configuration of confining strings is given by two individual minimal surfaces with no common points of intersection, which means that the average $\left\langle W\left(C_{1}\right) W\left(C_{2}\right)\right\rangle$ factorizes into $\left\langle W\left(C_{1}\right)\right\rangle\left\langle W\left(C_{2}\right)\right\rangle$ and no nonperturbative interaction takes place. This is in contrast to another approach, which is also based on the field correlator method, but follows a different logic. The strategy started in [9] has many successful phenomenological applications and modifications (see, e.g., [10]). The integration surfaces are taken in, e.g., [11] as individual minimal surfaces for each contour and all physical effects are due to the nonzero value of string thickness $T_{g}$ which brings about nonzero overlap between hadron Wilson loops. In other words, the expressions (31),(32) have a nontrivial limit if $T_{g} \rightarrow 0$, while the string tension $\sigma \sim\left\langle F^{2}\right\rangle T_{g}^{2}$ is kept fixed, in contrast to the corresponding expressions from [9-11] which vanish in this limit. Accordingly the hadron-hadron cross section in that approach is proportional to $T_{g}$ in the tenth power [10] and is very sensitive to the exact value of $T_{g}$. It is worth mentioning that the existing lattice calculations yield values in the range $0.35 \mathrm{fm}>T_{g}>0.1 \mathrm{fm}$ for different numbers of colors and light quark flavors (see [12] and references therein). However, the discussed difference between physical pictures is not just a question of numbers. We assume physical rearrangement of the confining strings at small distances (which could in principle be detected on the lattice for static contours), as happens, e.g., for soap films. For example, our answers (37) and (39) for parallel and oppositely oriented contours are drastically different, just because of the different geometry of the strings providing the minimal energy configuration. On the other hand, it remains to be studied which nonperturbative effect (the overlap due to the finite transverse size of the string or string rearrangement) plays the dominant role for the particular geometry of loops. We will argue that for the high-energy scattering case neither does, and the dominant contribution comes from the exchange by reggeized gluons in a confining background, i.e., Pomerons.

So, we adopt the following algorithm for computing the nonperturbative Wilson loop correlator. First, for a given geometry of the contours, we have to find the confining string configuration which gives the dominant contribution to the quantity $\left\langle W\left(C_{1}\right) W\left(C_{2}\right)\right\rangle$ (notice that there might be different surfaces for different representations that appear in the expansion of the product of fundamental Wilson loops). If the configuration providing the maximum coincides with individual minimal surfaces, we conclude that the nonperturbative interaction is absent. As the second step, we calculate the correlator $\chi^{(0)}\left(C_{1}, C_{2}\right)$ via Eq. (9) in terms of gaugeinvariant field correlators. We assume that the ensemble of correlators obeys the same hierarchy on this surface as it does on the minimal surface for a single Wilson loop, i.e., exhibits Gaussian dominance.

We keep generality at the moment and perform the vacuum averaging of Wilson loops, i.e., we explicitly calculate $\chi^{(0)}\left(C_{1}, C_{2}\right)$. Suppose that surfaces $S_{1}, S_{2}$ have already been chosen according to our criteria. For field-strength tensors belonging to surfaces $S_{1}$ and $S_{2}$ and gauge-transported to the same point $x_{0}$ we define

$$
(F d \sigma)^{(1)}(u)=F_{\mu \nu}\left(u, x_{0}\right) d \sigma_{\mu \nu}(u), \quad u \in S_{1},
$$

and analogously for the second surface. One can now write down the product of two Wilson loops in matrix form as

$$
\begin{aligned}
N_{c}^{2}\left\langle W\left(C_{1}\right) W\left(C_{2}\right)\right\rangle \\
=\left\langle\operatorname{Tr}_{1} \operatorname{Tr}_{2}, \mathrm{P}_{12}\left[\exp \left(i g \int_{S_{1}}(F d \sigma)^{(1)}(u)\right)\right]_{\alpha_{1} \beta_{1}}\right. \\
\left.\quad \times\left[\exp \left(i g \int_{S_{2}}(F d \sigma)^{(2)}(v)\right)\right]_{\alpha_{2} \beta_{2}}\right\rangle
\end{aligned}
$$

where the traces $\operatorname{Tr}_{1}$ and $\operatorname{Tr}_{2}$ go over indices carrying subscripts 1 and 2 , respectively, and the ordering operator $\mathrm{P}_{12}$ orders the products of matrices in a proper way according to the definition of the P exponent (4).

One can derive the following rule for vacuum averaging of several matrix operators, transported to one point (it is easy to show that these relations are gauge invariant under field-independent gauge transformations): 


$$
\begin{aligned}
\left\langle\left[F\left(u^{(1)}, x_{0}\right) \cdots F\left(u^{(k)}, x_{0}\right)\right]_{\alpha_{1} \beta_{1}}\left[F\left(v^{(1)}, x_{0}\right) \cdots F\left(v^{(m)}, x_{0}\right)\right]_{\alpha_{2} \beta_{2}}\right\rangle \\
=\frac{\delta_{\alpha_{1} \beta_{2}} \delta_{\alpha_{2} \beta_{1}}}{N_{c}^{2}-1}\left[\left\langle\operatorname{Tr}\left[F\left(u^{(1)}, x_{0}\right) \cdots F\left(u^{(k)}, x_{0}\right) F\left(v^{(1)}, x_{0}\right) \cdots F\left(v^{(m)}, x_{0}\right)\right]\right\rangle\right. \\
\left.\quad-\frac{1}{N_{c}}\left\langle\operatorname{Tr}\left[F\left(u^{(1)}, x_{0}\right) \cdots F\left(u^{(k)}, x_{0}\right)\right) \operatorname{Tr}\left(F\left(v^{(1)}, x_{0}\right) \cdots F\left(v^{(m)}, x_{0}\right)\right]\right\rangle\right] \\
\quad+\frac{\delta_{\alpha_{1} \beta_{1}} \delta_{\alpha_{2} \beta_{2}}}{N_{c}^{2}-1}\left[\left\langle\operatorname{Tr}\left[F\left(u^{(1)}, x_{0}\right) \cdots F\left(u^{(k)}, x_{0}\right) \operatorname{Tr}\left(F\left(v^{(1)}, x_{0}\right) \cdots F\left(v^{(m)}, x_{0}\right)\right]\right\rangle\right.\right. \\
\left.\quad-\frac{1}{N_{c}}\left\langle\operatorname{Tr}\left[F\left(u^{(1)}, x_{0}\right) \cdots F\left(u^{(k)}, x_{0}\right) F\left(v^{(1)}, x_{0}\right) \cdots F\left(v^{(m)}, x_{0}\right)\right]\right\rangle\right] .
\end{aligned}
$$

For the lowest Gaussian correlator when $k=m=1$ one has, from Eq. (14),

$$
\begin{aligned}
& \left\langle\left[F\left(u, x_{0}\right)\right]_{\alpha_{1} \beta_{1}}\left[F\left(v, x_{0}\right)\right]_{\alpha_{2} \beta_{2}}\right\rangle \\
& \quad=\frac{\left\langle\operatorname{Tr} F\left(u, x_{0}\right) F\left(v, x_{0}\right)\right\rangle}{N_{c}^{2}-1}\left(\delta_{\alpha_{1} \beta_{2}} \delta_{\alpha_{2} \beta_{1}}-\frac{1}{N_{c}} \delta_{\alpha_{1} \beta_{1}} \delta_{\alpha_{2} \beta_{2}}\right) .
\end{aligned}
$$

The above relations are valid in theories without global color symmetry breaking. Now one can proceed with the matrix cumulant expansion (see, e.g., [13]):

$$
\begin{aligned}
N_{c}^{2}\left\langle W\left(C_{1}\right) W\left(C_{2}\right)\right\rangle \\
\quad=\operatorname{Tr}_{1} \operatorname{Tr}_{2}, \mathrm{P}_{12} \exp \left(\sum_{n=1}^{\infty} \frac{(i g)^{n}}{n !}\langle\langle\widetilde{F}(1) \cdots \widetilde{F}(n)\rangle\rangle\right)
\end{aligned}
$$

where we use the notation

$$
\widetilde{F}(k)=\int_{S_{1}}(F d \sigma)^{(1)}\left(u^{(k)}\right)+\int_{S_{2}}(F d \sigma)^{(2)}\left(v^{(k)}\right) .
$$

Here the double brackets $\langle\langle\cdots\rangle\rangle$ denote irreducible correlators (see the definition in $[1,13])$. The quantity $\widetilde{F}(k)$ carries four independent fundamenatal color indices and the traces $\mathrm{Tr}_{1}, \mathrm{Tr}_{2}$ go over indices corresponding to $F^{(1)}$ and $F^{(2)}$, respectively.

Expression (16) provides the basis for our discussion. For a single loop the corresponding cluster expansion is given by

$$
\begin{aligned}
\left\langle W\left(C_{1}\right)\right\rangle= & \frac{1}{N_{c}} \operatorname{Tr}_{1}, \mathrm{P} \\
& \times \exp \left(\sum _ { n = 1 } ^ { \infty } \frac { ( i g ) ^ { n } } { n ! } \left\langle\left\langle\int_{S_{1}}(F d \sigma)^{(1)}\left(u^{(1)}\right) \cdots\right.\right.\right. \\
& \left.\left.\left.\times \int_{S_{1}}(F d \sigma)^{(1)}\left(u^{(n)}\right)\right\rangle\right)\right)
\end{aligned}
$$

sizes of the loops are larger than the gluon correlation length $T_{g}$ ) one gets

$$
\left\langle W\left(C_{1}\right)\right\rangle \propto \exp \left(-\sigma S_{1}^{\min }\right)
$$

The string tension $\sigma$ is given by

$$
\begin{aligned}
\sigma S_{1}^{\min }= & \frac{1}{2 N_{c}} \int_{S_{1}} d \sigma\left(u^{(1)}\right) \int_{S_{1}} d \sigma\left(u^{(2)}\right) \\
& \times\left\langle\operatorname{Tr} g F\left(u^{(1)}, x_{0}\right) g F\left(u^{(2)}, x_{0}\right)\right\rangle+\cdots
\end{aligned}
$$

where the ellipsis denotes higher non-Gaussian terms. As mentioned above, we do not take into account perimeter terms; it is implicitly supposed that all the considered loops are large enough in this sense. It is straightforward to rewrite Eq. (16) combined with Eqs. (18) and (14) in the following way:

$$
\begin{aligned}
& N_{c}^{2}\left\langle W\left(C_{1}\right) W\left(C_{2}\right)\right\rangle \\
& \quad=\operatorname{Tr}_{1} \operatorname{Tr}_{2} \exp \left[\hat{1} \cdot\left(\Lambda_{0}+\Lambda_{1}\right)+\hat{e} \cdot \Lambda_{e}\right]
\end{aligned}
$$

where

$$
\Lambda_{0}=-\sigma S_{1}-\sigma S_{2}
$$

and the terms $\Lambda_{1}$ and $\Lambda_{e}$ contain correlators of powers of $F$ defined on different surfaces and hence provide a contribution to $\chi^{(0)}$. They are as follows:

$$
\begin{aligned}
\Lambda_{1}= & \pm \frac{g^{2}}{N_{c}\left(N_{c}^{2}-1\right)} \int_{S_{1}} d \sigma\left(u^{(1)}\right) \\
& \times \int_{S_{2}} d \sigma\left(v^{(1)}\right)\left\langle\operatorname{Tr} F\left(u^{(1)}, x_{0}\right) F\left(v^{(1)}, x_{0}\right)\right\rangle+\cdots
\end{aligned}
$$

and in the area law regime (which means that the typical and 


$$
\begin{aligned}
\Lambda_{e}= & \mp \frac{g^{2}}{N_{c}^{2}-1} \int_{S_{1}} d \sigma\left(u^{(1)}\right) \\
& \times \int_{S_{2}} d \sigma\left(v^{(1)}\right)\left\langle\operatorname{Tr} F\left(u^{(1)}, x_{0}\right) F\left(v^{(1)}, x_{0}\right)\right\rangle+\cdots
\end{aligned}
$$

where the ellipses again denote higher non-Gaussian terms. The upper (lower) sign in the above expressions corresponds to the case of parallel (opposite) orientation of the contours $C_{1}$ and $C_{2}$ and surfaces $S_{1}$ and $S_{2}$ (we assume that the orientation of the surface is fixed by the orientation of its boundary contour).

The matrix structures $\hat{1}$ and $\hat{e}$ introduced above are given in index notation by

$$
\begin{aligned}
& {[\hat{1}]_{\alpha_{1} \beta_{1} ; \alpha_{2} \beta_{2}}=\delta_{\alpha_{1} \beta_{1}} \delta_{\alpha_{2} \beta_{2}}} \\
& {[\hat{e}]_{\alpha_{1} \beta_{1} ; \alpha_{2} \beta_{2}}=\delta_{\alpha_{1} \beta_{2}} \delta_{\alpha_{2} \beta_{1}} .}
\end{aligned}
$$

It can be checked that the matrices $\hat{1}$ and $\hat{e}$ obey the following algebra:

$$
\hat{e} \cdot \hat{1}=\hat{1} \cdot \hat{e}=\hat{e}, \quad \hat{1}^{2}=\hat{1}, \quad \hat{e}^{2}=\hat{1}
$$

for the case of parallel orientation of surfaces and

$$
\hat{e} \cdot \hat{1}=\hat{1} \cdot \hat{e}=\hat{e}, \quad \hat{1}^{2}=\hat{1}, \quad \hat{e}^{2}=N_{c} \hat{e}
$$

for antiparallel orientation. We also notice that in both cases $\operatorname{Tr}_{1} \operatorname{Tr}_{2} \hat{1}=N_{c}^{2}$ and $\operatorname{Tr}_{1} \operatorname{Tr}_{2} \hat{e}=N_{c}$.

With Eqs. (26), (27) at hand, we can easily compute Eq. (21). For parallel orientation of surfaces one gets

$$
\begin{aligned}
\chi^{(0)}\left(C_{1}, C_{2}\right)= & {\left[\frac{1}{2}\left(1-\frac{1}{N_{c}}\right) \exp \left(\Lambda_{0}+\Lambda_{1}-\Lambda_{e}\right)\right.} \\
& \left.+\frac{1}{2}\left(1+\frac{1}{N_{c}}\right) \exp \left(\Lambda_{0}+\Lambda_{1}+\Lambda_{e}\right)\right] \\
& -\exp \left(\Lambda_{0}^{\text {min }}\right)
\end{aligned}
$$

where the last term corresponds to the product of averages of two loops. For oppositely directed contours the result is

$$
\begin{aligned}
\chi^{(0)}\left(C_{1}, C_{2}\right)= & {\left[\frac{1}{N_{c}^{2}} \exp \left(\Lambda_{0}+\Lambda_{1}+N_{c} \Lambda_{e}\right)\right.} \\
& \left.+\left(1-\frac{1}{N_{c}^{2}}\right) \exp \left(\Lambda_{0}+\Lambda_{1}\right)\right]-\exp \left(\Lambda_{0}^{\min }\right) .
\end{aligned}
$$

Expressions (28),(29) together with the prescription for the choice of optimal integration surface provide the answer for the nonperturbative interaction term. Let us come to concrete examples and first consider the simplest possible case when $S_{1}=S_{1}^{\text {min }}, S_{2}=S_{2}^{\text {min }}$, and $S_{2}^{\text {min }} \subset S_{1}^{\text {min }}$ (see Fig. 1). As ex-

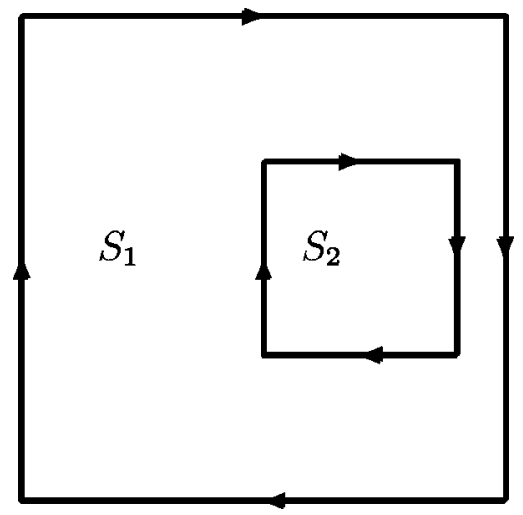

FIG. 1. Planar geometry of the Wilson loops for $S_{2}^{\min } \subset S_{1}^{\min }$ (the case of coinciding orientations).

plained in detail above, we always assume Gaussian dominance. One easily gets the following result for the contours with linear sizes greater than $T_{g}$ and omitting perimeter contributions:

$$
\Lambda_{1}= \pm \frac{2}{N_{c}^{2}-1} \sigma S_{2}^{\min }, \quad \Lambda_{e}=\mp \frac{2 N_{c}}{N_{c}^{2}-1} \sigma S_{2}^{\min }
$$

and Eqs. (28),(29) become

$$
\begin{aligned}
\chi^{(0)}\left(C_{1}, C_{2}\right) \\
=\exp \left(-\sigma S_{1}^{\text {min }}-\sigma S_{2}^{\text {min }}\right) \cdot\left[\frac{1}{2}\left(1-\frac{1}{N_{c}}\right) \exp \left(\frac{2 \sigma S_{2}^{\text {min }}}{N_{c}-1}\right)\right. \\
\left.\quad+\frac{1}{2}\left(1+\frac{1}{N_{c}}\right) \exp \left(-\frac{2 \sigma S_{2}^{\text {min }}}{N_{c}+1}\right)\right] \\
\quad-\exp \left(-\sigma S_{1}^{\text {min }}-\sigma S_{2}^{\text {min }}\right)
\end{aligned}
$$

for parallel orientations and

$$
\begin{aligned}
\chi^{(0)}\left(C_{1}, C_{2}\right) \\
=\exp \left(-\sigma S_{1}^{\text {min }}-\sigma S_{2}^{\text {min }}\right) \cdot\left[\frac{1}{N_{c}^{2}} \exp \left(2 \sigma S_{2}^{\text {min }}\right)\right. \\
\left.\quad+\left(1-\frac{1}{N_{c}^{2}}\right) \exp \left(-\frac{2 \sigma S_{2}^{\text {min }}}{N_{c}^{2}-1}\right)\right] \\
\quad-\exp \left(-\sigma S_{1}^{\text {min }}-\sigma S_{2}^{\text {min }}\right)
\end{aligned}
$$

for opposite orientations. It is worth recalling that $S_{1}^{\min }$ $>S_{2}^{\min }$ by our convention.

Notice that in the case of coinciding but oppositely directed contours (i.e., $C=C_{1}=\left[C_{2}\right]^{\dagger}$ ), expression (32) gives the Casimir scaled adjoint string tension:

$$
\sigma_{a d j}=\frac{2 N_{c}^{2}}{N_{c}^{2}-1} \sigma .
$$




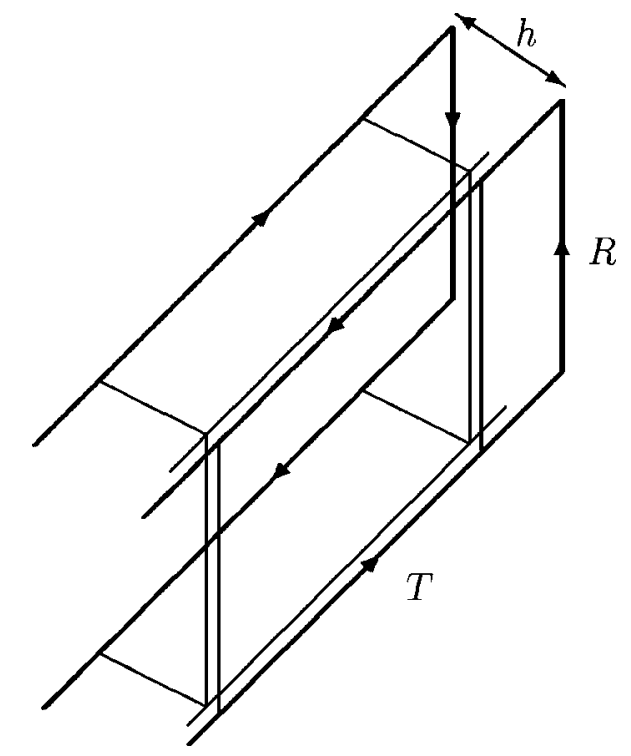

FIG. 2. Nonperturbative interaction of rectangular Wilson contours. The minimal string profile for the case of opposite orientations is given by "enveloping" geometry. Leading large area contribution corresponds to annihilation of fluxes along $R$.

This result is to be expected since, as already mentioned, Gaussian dominance yields Casimir scaling [7]. If both contours $C_{1}, C_{2}$ lie on the same plane and $C_{2}$ is inside $C_{1}$, the geometry becomes effectively two dimensional and the results (31),(32) coincide with formulas obtained in a slightly different way in [14]. The same expressions hold true in $(1+1)$-dimensional Yang-Mills theory, where one has just two-dimensional geometry and, on the other hand, an exact Gaussian picture. One can also look upon Eqs. (31),(32) as an algebraic rule for adding up parallel or antiparallel fundamental fluxes which illustrate the decomposition $\mathbf{3} \otimes \mathbf{3}=\overline{\mathbf{3}}$ $\oplus \mathbf{6}$ and $\mathbf{3} \otimes \overline{\mathbf{3}}=\mathbf{1} \oplus \mathbf{8}$, respectively, with the string tension in each representation given by the Casimir scaling law.

We are now interested in the case of contours separated by distances greater than $T_{g}$. By way of example let us calculate the purely nonperturbative correlator of two Wilson loops $\left\langle W\left(C_{1}\right) W\left(C_{2}\right)\right\rangle$ for simple rectangular geometry of the contours. We choose two rectangular contours $R \times T$ lying on parallel planes, at a distance $h$ from each other (see Figs. 2 and 3). We suppose that $T \gg R$ and will not take care of subleading $1 / T$ terms. If $h$ is of the order of $T_{g}$, one comes back to the case described by Eqs. (31),(32). We take the distance $h$ such that $R \geqslant h \geqslant T_{g}$, where the strings are supposed to rearrange themselves. Consider first the case of opposite loop orientations and let us examine different choices of surfaces. As a trivial example we might adopt the same choice as above, namely, $S_{1}=S_{1}^{\min }, S_{2}=S_{2}^{\min }$. The first term in square brackets in Eq. (29) contributes to $\left\langle W\left(C_{1}\right) W\left(C_{2}\right)\right\rangle$ as

$$
\frac{1}{N_{c}^{2}} \exp (-2 \sigma R T)
$$

as it should, since $\Lambda_{0}=\Lambda_{0}^{\text {min }}=\sigma S_{1}^{\min }+\sigma S_{2}^{\min }=2 \sigma R T$ and

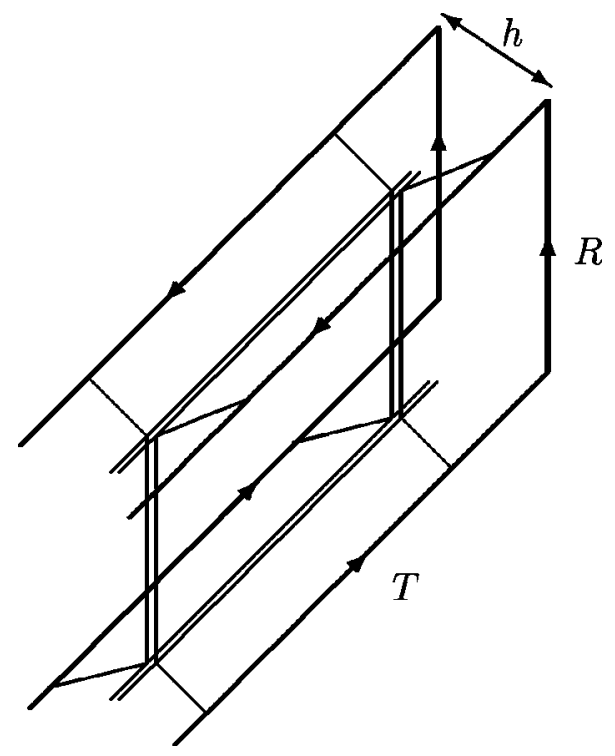

FIG. 3. Nonperturbative interaction of rectangular Wilson contours. The time slices of the minimal string profile for the case of parallel orientations are depicted. It is assumed that $N_{c}=3$. The leading large area contribution corresponds to a single fundamental flux in the $R$ direction.

$\Lambda_{1}=\Lambda_{e}=0$ for this choice of surfaces [up to exponentially small terms $\sim \exp \left(-2 h / T_{g}\right)$, which we always omit in this paper]. Correspondingly, the second term in square brackets in Eq. (29) would be

$$
\left(1-\frac{1}{N_{c}^{2}}\right) \exp (-2 \sigma R T)
$$

with the sum of the two giving the expected answer $\left\langle W\left(C_{1}\right)\right\rangle\left\langle W\left(C_{2}\right)\right\rangle=\exp (-2 \sigma R T)$ and hence $\chi^{(0)}=0$. One immediately sees that this choice is not optimal if $h$ is small. Instead, if we choose $S_{1}$ as a minimal enveloping surface with a boundary on $C_{1}$ and coinciding with $S_{2}=S_{2}^{\text {min }}$ inside $C_{2}$ (due to the apparent symmetry of our problem, one could of course easily interchange the indices 1 and 2), we get for the first term in square brackets in Eq. (29)

$$
\frac{1}{N_{c}^{2}} \exp (-2 \sigma h T)
$$

where $2 h T=S_{12}=S_{1}-S_{2}$. The second term contributes to $\left\langle W\left(C_{1}\right) W\left(C_{2}\right)\right\rangle$ as

$$
\left(1-\frac{1}{N_{c}^{2}}\right) \exp \left(-2 \sigma h T-\sigma_{a d j} R T\right)
$$

where $\sigma_{a d j}$ is given by Eq. (33) and this contribution is always subleading with respect to the former one if $R \gg h$ and $N_{c}$ is not exponentially large (see below). So these two different choices give different answers for the Wilson loop correlator:

$$
\left\langle W\left(C_{1}\right) W\left(C_{2}\right)\right\rangle=\exp (-2 \sigma R T)
$$


in the first case (two individual minimal surfaces) and

$$
\begin{aligned}
\left\langle W\left(C_{1}\right) W\left(C_{2}\right)\right\rangle= & \frac{1}{N_{c}^{2}} \exp (-2 \sigma h T)+\left(1-\frac{1}{N_{c}^{2}}\right) \\
& \times \exp \left(-2 \sigma h T-\sigma_{a d j} R T\right)
\end{aligned}
$$

in the second case (enveloping geometry). According to our criteria, the answer that is dominant should be chosen as correct, since it corresponds to the actual string configuration. It is seen that there is a critical distance between loops,

$$
h_{c r i t} \approx R-\frac{1}{\sigma T} \log N_{c},
$$

in our problem. ${ }^{2}$ For $h<h_{\text {crit }}$ confining strings rearrange themselves ${ }^{3}$ with respect to the noninteracting case, which is encoded in the expression (35). Correspondingly, one has nonzero $\chi^{(0)}$. For larger $h$ they do not interact and $\chi^{(0)}\left(C_{1}, C_{2}\right)$ vanishes. It is important that in our picture it happens dynamically; in particular, one cannot just naively take the large $N_{c}$ limit in Eqs. (28),(29). On the other hand, if $h$ is kept fixed, then it is clear from Eq. (36) that in the large $N_{c}$ limit $\chi^{(0)}$ should vanish. It is worth saying that with $T$ going to infinity in Eqs. (34),(35), $h_{c r i t}$ is increasing and approaching $R$; therefore for static loops the minimal energy state always wins eventually, as it should.

To summarize, the answer is given by

$$
\begin{aligned}
\chi^{(0)}= & \frac{1}{N_{c}^{2}} \exp (-2 \sigma h T)+\left(1-\frac{1}{N_{c}^{2}}\right) \exp \left(-2 \sigma h T-\sigma_{a d j} R T\right) \\
& -\exp (-2 \sigma R T)
\end{aligned}
$$

if $h<h_{\text {crit }}$ and $\chi^{(0)}=0$ if $h>h_{\text {crit }}$. We now come to the case of parallel orientations. Let us consider the two geometries analyzed above. The case of individual minimal surfaces is identical for parallel and opposite orientations and has just been considered. The enveloping geometry gives for the first term in square brackets in Eq. (28)

$$
\frac{1}{2}\left(1-\frac{1}{N_{c}}\right) \exp \left(-2 \sigma h T-\frac{2 \sigma R T\left(N_{c}-2\right)}{N_{c}-1}\right),
$$

while the second term becomes

$$
\frac{1}{2}\left(1+\frac{1}{N_{c}}\right) \exp \left(-2 \sigma h T-\frac{2 \sigma R T\left(N_{c}+2\right)}{N_{c}+1}\right)
$$

and is always subleading. In the particular case $N_{c}=3$ the former term corresponds to the creation of the string in $\overline{\mathbf{3}}$ representation of the length $R$ which has the same tension as the fundamental string, while the second term describes the

${ }^{2}$ For Abelian confining strings including soap films the last term in Eq. (36) is absent.

${ }^{3}$ See also [15], where static multiquark interactions were studied in the strong coupling expansion regime. sextet representation string formation $\mathbf{6}=\operatorname{Symm}\{\mathbf{3} \otimes \mathbf{3}\}$ with tension $2\left(N_{c}+2\right) /\left(N_{c}+1\right)=2.5$ times larger than the fundamental one. In this particular case of $N_{c}=3$ one can additionally consider a double Y-shape profile, shown in Fig. 3, with the result for the first term in square brackets in Eq. (28)

$$
\frac{1}{3} \exp (-\sqrt{3} \sigma h T-\sigma R T)
$$

Since $\sqrt{3}<2$, this term is seen to be dominant over the enveloping geometry in the $N_{c}=3$ case.

As in the opposite orientation case there appears a critical length $h_{c r i t}$ which for $N_{c}=3$ is given by

$$
h_{\text {crit }}=\frac{1}{\sqrt{3}}\left(R-\frac{\log 3}{\sigma T}\right) .
$$

The expression for $\chi^{(0)}$ becomes $\left(N_{c}=3\right)$

$$
\begin{aligned}
\chi^{(0)}\left(C_{1}, C_{2}\right)= & \exp \left(-\sigma S_{12}\right) \cdot\left[\frac{1}{3} \exp \left(-\sigma S_{3}\right)\right. \\
& \left.+\frac{2}{3} \exp \left(-\frac{5 \sigma S_{3}}{2}\right)\right]-\exp (-2 \sigma R T)
\end{aligned}
$$

where $S_{12}$ represents the boundary surface $S_{12}=4 h T / \sqrt{3}$ and $S_{3}=(R-h / \sqrt{3}) T$ is the common part of $S_{1}$ and $S_{2}$. Notice that $l_{\text {crit }}=R-h_{\text {crit }} / \sqrt{3}>0$; therefore one is never in the situation of $S_{3}$ shrinking to zero.

The physical picture is the same as for opposite orientations-at small distances $h, h_{\text {crit }}>h \geqslant T_{g}$, the preferable string configuration is given by the double Y-shape profile shown in Fig. 3 while at larger distances there is no common string state formation and hence the nonperturbative interaction is absent, $\chi^{(0)}\left(C_{1}, C_{2}\right)=0$.

To conclude this section let us say a few words about the case of distant loops. If contours are distant from each other, it is always preferable to deform $S_{12}$ to two disks, corresponding to minimal surfaces for each contour plus whatever thin tube connects these surfaces through the point $x_{0}$, thus reducing $\chi^{(0)}\left(C_{1}, C_{2}\right)$ to zero. This is a sign that the purely nonperturbative contribution vanishes, and one should consider next terms in the expansion (8), namely, $\chi^{(4)}\left(C_{1}, C_{2}\right)$. It is important to realize that the surface entering Eq. (9) is not dynamical, ${ }^{4}$ which is reflected in the possibility of infinite squeezing of the tube connecting two distant minimal surfaces. The situation changes, however, when one includes perturbative gluons, propagating inside its wall and forming a physical glueball state in this way. It is actually this glueball exchange mechanism which corresponds to the term

\footnotetext{
${ }^{4}$ It has come from the Stokes theorem and therefore could be arbitrary, subject to our principle of minimal action.
} 
$\chi^{(4)}\left(C_{1}, C_{2}\right)$ when the background field is taken into account. We study this term in the next secton.

\section{GLUEBALL EXCHANGE INTERACTION}

We now turn to the term $\chi^{(4)}\left(C_{1}, C_{2}\right)$ in the expansion (8). We shall see that in the absence of a nonperturbative background this term reduces to the purely perturbative twogluon exchange term suggested in [16] as a basic element of Pomeron exchange. To be more precise, let us consider first the case of no background fields. In the Feynman gauge for $a_{\mu}$, one has in the lowest order

$$
\left\langle a_{\mu}^{a}(x) a_{\nu}^{b}(y)\right\rangle=\delta_{\mu \nu} \delta^{a b} \frac{1}{4 \pi^{2}(x-y)^{2}} .
$$

Our expression for $\chi^{(4)}$ will have the same form as Eq. (21), where one should keep only the nonperturbative background field $B_{\mu}$ in diagonal correlators $\left\langle\operatorname{Tr} F^{1} F^{1}\right\rangle$ while terms proportional to $\left\langle\operatorname{Tr} F^{1} F^{2}\right\rangle$ contain only perturbative exchange. These perturbative exchanges are modified, however, by the presence of the nonperturbative background. To take it into account, one has to perform averaging in two steps: first in the valence (perturbative) field $a_{\mu}$ and second in the background field $B_{\mu}$ :

$$
\begin{aligned}
\left\langle W\left(C_{1}\right) W\left(C_{2}\right)\right\rangle= & \frac{g^{4}}{N_{c}^{2}} \operatorname{Tr}_{1} \operatorname{Tr}_{2}, \mathrm{P}_{12} \int d x_{\mu_{1}}^{(1)} \int d x_{\mu_{2}}^{(2)} \int d y_{\nu_{1}}^{(1)} \int d y_{\nu_{2}}^{(2)} \cdot \Phi_{C_{1}}\left(x^{(2)}, x^{(1)}\right) t^{a_{1}} \Phi_{C_{1}}\left(x^{(1)}, x^{(2)}\right) t^{a_{2}} \Phi_{C_{2}} \\
& \times\left(y^{(2)}, y^{(1)}\right) t^{b_{1}} \Phi_{C_{2}}\left(y^{(1)}, y^{(2)}\right) t^{b_{1}} \cdot\left[\left\langle a\left(x^{(1)}\right) a\left(y^{(1)}\right)\right\rangle\left\langle a\left(x^{(2)}\right) a\left(y^{(2)}\right)\right\rangle+\left\langle a\left(x^{(1)}\right) a\left(y^{(2)}\right)\right\rangle\right. \\
& \left.\times\left\langle a\left(x^{(2)}\right) a\left(y^{(1)}\right)\right\rangle\right]
\end{aligned}
$$

where the shorthand notation $a\left(x^{(1)}\right) \equiv a_{\mu_{1}}^{a_{1}}\left(x^{(1)}\right)$ was used. The coordinates $x^{(1)}, x^{(2)}$ are ordered along the contour $C_{1}$ and $y^{(1)}, y^{(2)}$ are ordered along the contour $C_{2}$. Notice also the gauge invariance of Eq. (41) due to the transformation law (2).

Before proceeding further one is to define the dependence of $\left\langle a_{\mu}(x) a_{\nu}(y)\right\rangle$ on background fields. To this end it is convenient to use the Feynman-Schwinger representation for the gluon Green's function [3] and represent it as

$$
\begin{aligned}
G_{\mu \nu}^{a b} & =\left\langle a_{\mu}^{a}(x) a_{\nu}^{b}(y)\right\rangle \\
& =\int_{0}^{\infty} d s(\mathcal{D} z)_{x y} \exp \left(-K_{0}\right) \Phi_{\mu \nu}^{a b}(x, y)
\end{aligned}
$$

where

$$
K_{0}=\frac{1}{4} \int_{0}^{\infty} d \tau\left(\frac{d z_{\mu}}{d \tau}\right)^{2}
$$

and

$$
\begin{aligned}
\Phi_{\mu \nu}^{a b}(x, y)= & {\left[\mathrm{P}_{F} \mathrm{P}_{A} \exp \left(i g \int_{y}^{x} A_{\mu}(z) d z_{\mu}\right)\right.} \\
& \left.\times \exp \left(2 g \int_{0}^{s} d \tau F(z(\tau))\right)\right]_{\mu \nu}^{a b}
\end{aligned}
$$

and $a, b$ are adjoint color indices, whereas $\mu, \nu$ are Lorentz indices, i.e., $[F]_{\mu \nu}^{a b}=-i F_{\mu \nu}^{c} f^{a b c}$.

To understand better the topology of the resulting construction, it is useful to consider the large $N_{c}$ limit. One can write in this limit for the adjoint phase factors in Eq. (42)

$$
\begin{aligned}
& {\left[t^{a}\right]_{\alpha \beta} \Phi^{a b}(x, y)\left[t^{b}\right]_{\gamma \delta}} \\
& \quad=\mathrm{P}_{A} \exp \left(i g \int_{y}^{x} \hat{A}_{\lambda} d z_{\lambda}\right)^{a b}\left[t^{a}\right]_{\alpha \beta}\left[t^{b}\right]_{\gamma \delta} \\
& \quad=\Phi_{\alpha \delta}(x, y) \Phi_{\gamma \beta}(y, x)+\mathcal{O}\left(\frac{1}{N_{c}}\right)
\end{aligned}
$$

where $\Phi_{\alpha \delta}(x, y)$ is a parallel transporter in the fundamental representation. The expression (43) exemplifies the well known 't Hooft rule for replacing the gluon line by a double adjoint in the large $N_{c}$ limit. Inserting Eq. (43) into Eq. (41), one obviously obtains two new Wilson loops $C_{12}$ and $C_{12}{ }^{\prime}$ instead of the previous $C_{1}$ and $C_{2}$ : each initial loop is now divided by two gluon emissions/absorptions into two arcs which are connected by double lines of gluon propagators (see Figs. 4 and 5). At small $N_{c}$ this construction goes over into that of two fundamental loops $C_{1}$ and $C_{2}$ connected by

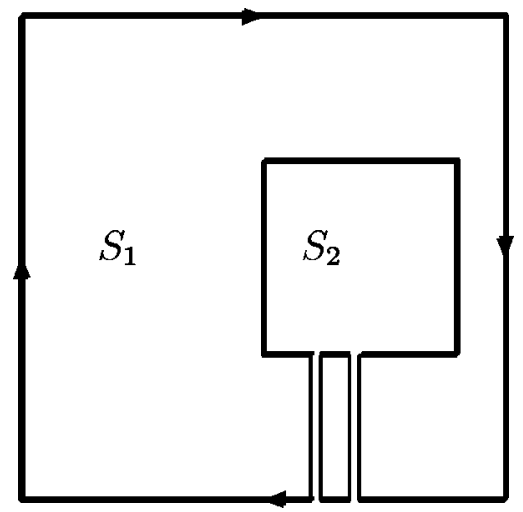

FIG. 4. Dominant two-gluon glueball term responsible for perturbative interaction with the two-dimensional geometry of Fig. 1. 


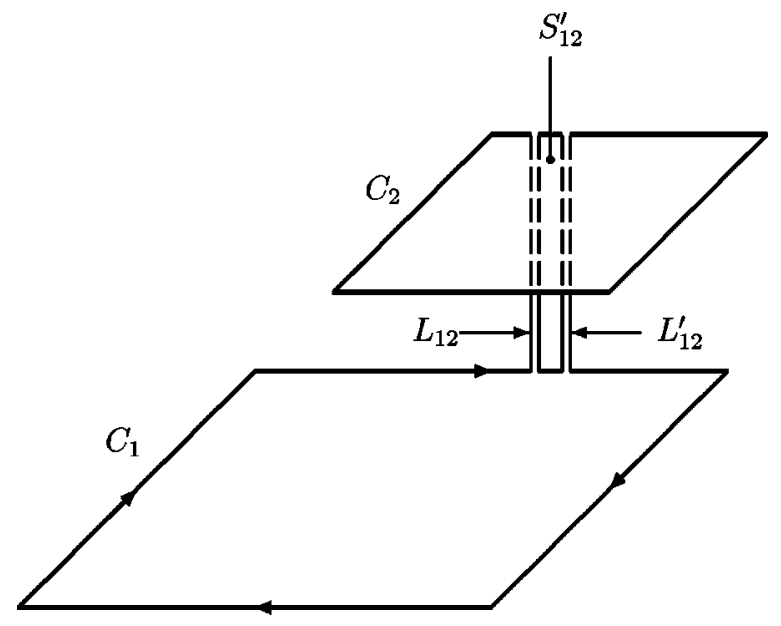

FIG. 5. Dominant two-gluon glueball term responsible for perturbative interaction in nonperturbative background at large distances. Gluon propagator lines are replaced by double fundamental lines in large $N_{c}$ limit.

two adjoint lines and the final result will amount to replacing a double fundamental string worldsheet by one adjoint string wordsheet. In terms of string tensions it corresponds to replacement of $2 \sigma$ by $9 \sigma / 4$ in the Gaussian approximation. We will keep the large $N_{c}$ limit and replacement (43) in what follows.

The averaging over background fields leads to the following result:

$$
\begin{aligned}
\left\langle\left\langle W\left(C_{1}\right) W\left(C_{2}\right)\right\rangle_{a}^{(4)}\right\rangle_{B}= & \chi^{(4)}\left(C_{1}, C_{2}\right) \\
= & \frac{\tilde{g}^{4}}{N_{c}^{2}} \int_{L_{12}} d s_{1} \mathcal{D} z^{(1)} \int_{L_{12}{ }^{\prime}} d s_{2} \mathcal{D} z^{(2)} \\
& \times \exp \left(-K_{0}^{(1)}-K_{0}^{(2)}\right) \\
& \times\left\langle W\left(C_{12}\right) W\left(C_{12}^{\prime}\right)\right\rangle
\end{aligned}
$$

where $\tilde{g}^{2}=g^{2} N_{c}$. Here the contours $C_{12}$ and $C_{12}{ }^{\prime}$ comprise pieces of $C_{1}$ and $C_{2}$ connected by two double fundamental lines $L_{12}$ and $L_{12}{ }^{\prime}$. It is understood that the surfaces $S_{12}, S_{12}{ }^{\prime}$ are subject to our general assumption about minimal action. This gives different forms depending on the distance between the original loops $C_{1}$ and $C_{2}$ (see below). It is also understood that the gluon spin operators $2 g F(z)$ are to be placed on the gluon trajectories $L_{12}$ and $L_{12}{ }^{\prime}$ in accordance with Eq. (42). It will produce gluon spin interaction terms which influence the glueball Green's function; to simplify discussion we omit these terms at the moment.

Now we can use the large $N_{c}$ factorization property for the product $\left\langle W\left(C_{12}\right) W\left(C_{12}{ }^{\prime}\right)\right\rangle$ and use area law asymptotics for each piece, i.e., for surfaces $S_{12}$ and $S_{12}{ }^{\prime}$. One obtains

$$
\begin{aligned}
\chi^{(4)}\left(C_{1}, C_{2}\right)= & \frac{\widetilde{g}^{4}}{N_{c}^{2}} \int_{L_{12}} d s_{1} \mathcal{D} z^{(1)} \int_{L_{12}} d s_{2} \mathcal{D} z^{(2)} \\
& \times \exp \left(-K_{0}^{(1)}-K_{0}^{(2)}\right) \exp \left[-\sigma\left(S_{12}+S_{12}^{\prime}\right)\right] .
\end{aligned}
$$

To define the profiles of the surfaces we shall use the same principle outlined above, i.e., we require the effective value of the area $\left\langle S_{12}+S_{12}{ }^{\prime}\right\rangle$ averaged over possible gluon trajectories $L_{12}$ and $L_{12}{ }^{\prime}$ to be minimal. The result will of course strongly depend on the relative positions and orientations of the contours $C_{1}$ and $C_{2}$. In the first case when both loops lie on the same plane and $C_{2}$ is entirely inside $C_{1}$, it is clear that the sum $S_{12}+S_{12}$ ' does not depend on trajectories $L_{12}$ and $L_{12}{ }^{\prime}$, and one has $S_{12}+S_{12}{ }^{\prime}=S_{1}-S_{2}$. Thus one obtains effectively the surface $S_{1}$ with the hole due to $C_{2}$, i.e., a construction which has already appeared in purely nonperturbative term for oppositely oriented contours $C_{1}, C_{2}$, but now with two valence gluons connecting contours $C_{1}$ and $C_{2}$ (see Fig. 4).

However, for large enough distances the true minimum of $S_{12}+S_{12}{ }^{\prime}$ is reached by another construction-when the two contours $C_{1}$ and $C_{2}$ are connected by a narrow strip formed by the trajectories $L_{12}$ and $L_{12}{ }^{\prime}$ with the double (adjoint) string worldsheet between them (Fig. 5). This narrow strip is nothing but the glueball Green's function and the width of the strip is equal to the average size of the lowest-mass glueball, i.e., around $0.5 \mathrm{fm}$. Notice that due to the kinetic terms in Eq. (45) this strip is dynamical (contrary to the nonperturbative case) and cannot be shrunk. This is a typical construction for the high-energy scattering amplitude when the glueball exchange diagram is gradually replaced by the glueball Regge trajectory exchange, i.e., by Pomeron exchange, which persists to larger experimentally accessible energies.

To demonstrate that explicitly, one should rewrite the expression (45) directly in terms of the glueball Green's function:

$$
\begin{aligned}
\frac{\chi^{(4)}\left(C_{1}, C_{2}\right)}{\left\langle W\left(C_{1}\right) W\left(C_{2}\right)\right\rangle}= & \mathrm{P}_{12} \int_{C_{1}} d x_{\mu_{1}}^{(1)} \int_{C_{1}} d x_{\mu_{2}}^{(2)} \int_{C_{2}} d y_{\nu_{1}}^{(1)} \\
& \times \int_{C_{2}} d y_{\nu_{2}}^{(2)}\left[G_{\mu_{1} \mu_{2}}^{\nu_{1} \nu_{2}}\left(x^{(1)}, x^{(2)} \mid y^{(1)}, y^{(2)}\right)\right. \\
& \left.+\left(y^{(1)} \leftrightarrow y^{(2)}\right)\right]
\end{aligned}
$$

where $G_{\mu_{1} \mu_{2}}^{\nu_{1} \nu_{2}}\left(x^{(1)}, x^{(2)} \mid y^{(1)}, y^{(2)}\right)$ is the two-gluon glueball Green's function, describing propagation from points $x^{(1)}, x^{(2)}$ to $y^{(1)}, y^{(2)}$, which has the Feynman-Schwinger representation as in Eq. (45). The spectrum of this Green's function (with spin terms included) was computed analytically in [17].

When all points are close to each other, i.e.,

$$
\left|x^{(i)}-x^{(j)}\right| \ll T_{g}, \quad\left|x^{(i)}-y^{(j)}\right| \ll T_{g},
$$

one can replace the glueball Green's function by the product of free gluon propagators

$$
\begin{aligned}
& G_{\mu_{1} \mu_{2}}^{\nu_{1} \nu_{2}}\left(x^{(1)}, x^{(2)} \mid y^{(1)}, y^{(2)}\right) \\
& \quad \sim g^{4} \frac{\delta_{\mu_{1} \nu_{1}} \delta_{\mu_{2} \nu_{2}}}{(2 \pi)^{4}\left(x^{(1)}-y^{(1)}\right)^{2}\left(x^{(2)}-y^{(2)}\right)^{2}} .
\end{aligned}
$$


Another asymptotics is available when both $\left|x^{(i)}-y^{(j)}\right|$ are large; then the spectral decomposition is possible:

$$
\begin{aligned}
G_{\mu_{1} \mu_{2}}^{\nu_{1} \nu_{2}}\left(x^{(1)}, x^{(2)} \mid y^{(1)}, y^{(2)}\right) & \\
& \sim \sum_{n} \Psi_{\mu_{1} \mu_{2}}^{(n)}\left(x^{(1)}, x^{(2)}\right) \Psi_{\nu_{1} \nu_{2}}^{(n)}{ }^{\dagger}\left(y^{(1)}, y^{(2)}\right) \\
& \cdot \exp \left(-M_{n} \cdot\left|\frac{x^{(1)}+x^{(2)}}{2}-\frac{y^{(1)}+y^{(2)}}{2}\right|\right) .
\end{aligned}
$$

Since the lowest glueball is rather heavy, $M_{0} \approx 1.5 \mathrm{GeV}$, one expects a fast decrease of $\chi^{(4)}$ when the distance between loops is growing:

$$
\chi^{(4)}(h) \sim \exp \left(-M_{0}|h|\right) .
$$

The situation is qualitatively similar to the one studied in $[18,19]$, where the gluon was assumed to have effective mass $m_{g} \sim 0.9 \mathrm{GeV}$. One expects a dipole-dipole cross section around a few millibarns in this case, when $\alpha_{s}$ is of the order of 1 . To obtain realistic large hadron-hadron scattering one needs the glueball exchange to be reggeized, in which case the radius of interaction grows logarithmically [20]. In the particular case of the Balitskii-Fadin-Kuraev-Lipatov (BFKL) Pomeron this picture was studied in [21]. Our picture differs from that of BFKL, since the nonperturbative background is taken into account. For example, in the problem of high-energy forward onium-onium scattering the interaction time between particles at high energies is much smaller than the typical interaction time for quarks inside onium, and hence one can consider the onium in this process as a free quark-antiquark pair (see, e.g., [18]). The small radius of the onium compared with typical transversal length scales of the problem dictates $\chi^{(4)}$ dominance over $\chi^{(0)}$ in the problem since possible nonperturbative string configurations which could contribute to $\chi^{(0)}$ are strongly suppressed over individual minimal noninteracting strings.

Since it is more convenient to study the scattering of systems in given quantum states rather than the scattering of Wilson loops, we switch to spectral decomposition and take only one term, corresponding to the scattering of particular states. The resulting expression coincides (up to a normalization factor) with the scattering amplitude (see, e.g., [22]). Since in the Feynman gauge for the field $a_{\mu}^{a}$ we have

$$
\begin{aligned}
& G_{\mu_{1} \mu_{2}}^{\nu_{1} \nu_{2}}\left(x^{(1)}, x^{(2)} \mid y^{(1)}, y^{(2)}\right) \\
& \quad=\delta_{\mu_{1} \nu_{1}} \delta_{\mu_{2} \nu_{2}} G^{(2)}\left(x^{(1)}, x^{(2)} \mid y^{(1)}, y^{(2)}\right)
\end{aligned}
$$

the answer can be straightforwardly obtained using the same strategy as in [18]:

$$
\begin{aligned}
T_{\text {for } w}= & i \int d^{2} \rho_{1} \int d^{2} \rho_{2} \int_{0}^{1} d z_{1} \int_{0}^{1} d z_{2}\left|\psi\left(\rho_{1}, z_{1}\right)\right|^{2} \\
& \times\left|\psi\left(\rho_{2}, z_{2}\right)\right|^{2} F\left(\rho_{1}, \rho_{2}, Q=0\right)
\end{aligned}
$$

where

$$
\begin{aligned}
& F\left(\rho_{1}, \rho_{2}, Q=0\right) \\
& =\frac{N_{c}^{2}-1}{32 \pi^{2} N_{c}^{2}} \int d^{2} k G^{(2)}(k, Q=0) \cdot\left[2-\exp \left(-i k \rho_{1}\right)\right. \\
& \left.\quad-\exp \left(i k \rho_{1}\right)\right]\left[2-\exp \left(-i k \rho_{2}\right)-\exp \left(i k \rho_{2}\right)\right] .
\end{aligned}
$$

In the above expression $G^{(2)}(k, Q)$ is the Fourier transform of Eq. (49) with respect to total momentum $p_{1}+p_{2}=Q$ and relative momentum $p_{1}-p_{2}=Q-2 k$; the former is equal to zero for the forward scattering amplitude [we also suppose in Eq. (51) vanishing transverse momenta of onia]. The mixed representation wave function $\psi\left(\rho_{1}, z_{1}\right)$ defined on the light cone describes the state of the color dipole with transverse size $\rho_{1}$ and the fraction of total onium light cone momentum $p_{1}^{+}$carried by the quark $z_{1}$. We omit spinor indices, assuming proper summation over them. If one "turns off" confinement (i.e., in our formalism put the confining background field to zero everywhere), the Green's function in the leading order of perturbation theory will be just a product of two gluon propagators:

$$
G_{\text {free }}^{(2)}(k, Q)=\frac{g^{4}}{(Q-k)^{2} k^{2}},
$$

and inserting Eq. (52) into Eq. (51) one returns to the results of [18]. One would expect that the effects of confinement suppress the amplitude [18] in two different ways: first, because of the mass gap (and actually quite a large mass even for the lightest glueball), and, second, due to the fast decrease of the glueball wave function at large relative distances [see Eq. (48)]. These properties solve the artifact of color van der Waals forces appearing in the purely perturbative dipole-dipole interaction.

\section{CONCLUSIONS}

In the present paper we discussed interactions of Wilson loops in confining theory, having in mind gluodynamics as a concrete example. The effects of confinement were taken into account in the formalism of perturbation theory in a confining background. We described the background by a gauge-invariant Gaussian correlator with small correlation length, which is supported by lattice and analytic calculations. Two main physically different mechanisms of interaction were analyzed. The first one, which we call nonperturbative, refers to the process of a confining string rearrangement, which can be energetically preferable for particular geometries of the contours. In this way a common surface of two contours $C_{1}, C_{2}$ is created and in the case of opposite orientation this surface is a ring between $C_{1}$ and $C_{2}$ (with a hole inside the smaller loop). This mechanism has a direct classical analogue in soap films, while for parallel orientation non-Abelian properties of Wilson loops lead to a nonclassical configuration with the same ring but the hole filled by the film. The second mechanism arises due to twogluon exchange between loops and the corresponding amplitude is $O\left(g^{4}\right)$. In the confining background and at large $N_{c}$ this simple picture of two contours connected by two gluon lines is transformed into a new geometry of two new com- 
posite loops, as shown in Figs. 4 and 5. As a result one has two types of surface configurations-for small and for large separations between minimal surfaces $S_{1}$ and $S_{2}$, shown respectively in Fig. 4 and Fig. 5. We have briefly argued that the configuration generic for the scattering corresponds to Fig. 5, and reduces to the (reggeized) glueball exchanges between loops, while for the case of decay and sea quark loop effects both nonperturbative and perturbative mechanisms are important with small separation between $S_{1}$ and $S_{2}$. The results obtained in the paper provide a basis for a systematic development both in the direction of nonperturbative approach to hadron scattering and in the direction of the theory of strong hadron decays.

\section{ACKNOWLEDGMENTS}

The authors acknowledge support from the grants RFFI00-02-17836 and RFFI-00-15-96786 and from the grant INTAS 00-110. V.Sh. is grateful to the foundation "Fundamenteel Onderzoek der Materie" (FOM), which is financially supported by the Dutch National Science Foundation (NWO). V.Sh. also acknowledges support from the grant RFFI-01-02-06284. The work of Yu.S. was supported by DOE contract DE-AC05-84ER40150 under which SURA operates the Thomas Jefferson National Accelerator Facility, and by the grant INTAS 00-366.
[1] H. G. Dosch, V. Shevchenko, and Yu. Simonov, hep-ph/0007223.

[2] K. G. Wilson, Phys. Rev. D 10, 2445 (1974).

[3] Yu. A. Simonov and J. A. Tjon, Ann. Phys. (N.Y.) 228, 1 (1993); hep-ph/0201005.

[4] B. S. De Witt, Phys. Rev. 162, 1195 (1967); 162, 1239 (1967); J. Honerkamp, Nucl. Phys. B36, 130 (1972); L. F. Abbott, ibid. B185, 189 (1981).

[5] Y. A. Simonov, Nucl. Phys. B307, 512 (1988); Yad. Fiz. 58, 113 (1995) [Phys. At. Nucl. 58, 107 (1995)]; in Lecture Notes in Physics, edited by H. Latal and W. Schwinger (SpringerVerlag, Berlin, 1996), Vol. 479, p. 139.

[6] S. V. Ivanov and G. P. Korchemsky, Phys. Lett. 154B, 197 (1985); S. V. Ivanov, G. P. Korchemsky, and A. V. Radyushkin, Yad. Fiz. 44, 230 (1986) [Sov. J. Nucl. Phys. 44, 145 (1986)]; V. I. Shevchenko and Y. A. Simonov, Phys. Lett. B 437, 146 (1998); L. Lukaszuk, E. Leader, and A. Johansen, Nucl. Phys. B562, 291 (1999).

[7] Y. A. Simonov, JETP Lett. 71, 127 (2000); V. I. Shevchenko and Y. A. Simonov, Phys. Rev. Lett. 85, 1811 (2000); hep-ph/ 0104135.

[8] H. G. Dosch, Phys. Lett. B 190, 177 (1987); H. G. Dosch and Y. A. Simonov, ibid. 205, 339 (1988); Y. A. Simonov, Nucl. Phys. B307, 512 (1988).

[9] A. Kramer and H. G. Dosch, Phys. Lett. B 252, 669 (1990); O. Nachtmann, Ann. Phys. (N.Y.) 209, 436 (1991).
[10] H. G. Dosch, E. Ferreira, and A. Kramer, Phys. Rev. D 50, 1992 (1994); O. Nachtmann, hep-ph/9609365.

[11] A. I. Shoshi, F. D. Steffen, and H. J. Pirner, hep-ph/0202012.

[12] A. Di Giacomo and E. Meggiolaro, Phys. Lett. B 537, 173 (2002); A. Di Giacomo, Nucl. Phys. B (Proc. Suppl.) 108, 21 (2002).

[13] N. G. van Kampen, Stochastic Processes in Physics and Chemistry (North-Holland, Amsterdam, 1984).

[14] A. Y. Dubin and Y. S. Kalashnikova, Yad. Fiz. 58, 2078 (1995) [Phys. At. Nucl. 58, 1967 (1995)].

[15] H. G. Dosch, Phys. Rev. D 28, 412 (1983).

[16] F. E. Low, Phys. Rev. D 12, 163 (1975); S. Nussinov, Phys. Rev. Lett. 34, 1286 (1975).

[17] A. B. Kaidalov and Yu. A. Simonov, Phys. Lett. B 477, 163 (2000).

[18] A. Mueller and B. Patel, Nucl. Phys. B425, 471 (1994); A. Bialas, R. Peschanski, and Ch. Royon, Phys. Rev. D 57, 6899 (1998).

[19] N. Nikolaev and B. Zakharov, Z. Phys. C 49, 607 (1991); N. Nikolaev, B. Zakharov, and V. Zoller, Phys. Lett. B 328, 486 (1994).

[20] V. Gribov, Zh. Éksp. Teor. Fiz. 41, 667 (1961); G. Chew and S. C. Frautschi, Phys. Rev. Lett. 7, 394 (1961).

[21] E. A. Kuraev, L. N. Lipatov, and V. S. Fadin, Zh. Éksp. Teor. Fiz. 72, 377 (1977) [Sov. Phys. JETP 45, 199 (1977)]; Ya. Ya. Balitsky and L. N. Lipatov, Sov. J. Nucl. Phys. 28, 822 (1978).

[22] M. E. Peskin, Nucl. Phys. B156, 365 (1979). 